\title{
Yüksek Mertebeden Euler-Lagrange Denklemlerinin İndirgemeleri ve Hamilton Analizleri
}

\author{
Reduction of Higher Order Euler-Lagrange Equations and Their Hamiltonian Analysis
}

\author{
Filiz ÇAĞATAY UÇGUN 1 (10) \\ ${ }^{1}$ Maltepe Üniversitesi, Mühendislik ve Doğa Bilimleri Fakültesi, Yazılım Mühendisliği, Marmara Eğitim Köyü 34857 \\ Maltepe / ISTANBUL
}

\begin{abstract}
$\ddot{O} \mathbf{z}$
İkinci mertebeden türevlere bağımlı Lagrange fonksiyonlarını yeni koordinat tanımlayarak ve/veya Lagrange çarpımı kullanarak birinci mertebeden türevlere bağımlı hale getirmek mümkündür. İndirgeme olarak tanımlayacağımız bu süreç için literatürde verilen 3 yöntem karşılaştırılmıştır. Bu yöntemler ışı̆̆ında, yozlaşmama şartını sağlayan ikinci derece Lagrange fonksiyonlarının Hamilton analizi, Dirac-Bergmann metodu kullanılarak başarılmıştır. Tüm bu teorik inşalara örnek olarak Chern-Simons teorisi bünyesindeki yozlaşmama şartını sağlayan Chiral salınacı örneği detaylı olarak incelenmiştir.
\end{abstract}

Anahtar Kelimeler: Chiral Salınac1, Schmidt metodu, İkinci mertebe Lagrange fonksiyonları, Dirac-Bergmann algoritmas1

\begin{abstract}
It is possible to write a second order Lagrangian in a first order form by defining new coordinates or/and by introducing Lagrange multipliers. We call this as reductions procedure. In this work, we present and compare three methods of this reduction. In the light of these methods, Hamiltonian analysis of degenerate second order Lagrangians are achieved by using the Dirac-Bergmann algorithm. In order to illustrate these theoritical constructions, we study the degenerate Chiral oscillator in detail, within the Chern-Simons framework.
\end{abstract}

Keywords. Chiral Oscillator, Schmidt' method, Second Order Lagrangians, Dirac-Bergmann algorithm.

\section{I.GİRIŞ}

Fiziksel sistemler temel olarak Lagrange ve Hamilton olmak üzere iki biçimde ifade edilirler [1]. Lagrange denklemleri hızfaz uzayında tanımlı bir Lagrange fonksiyonları ile, Hamilton denklemleri ise momentum-faz uzayı üzerindeki Hamilton fonksiyonları ile tanımlanırlar. Yozlaşmama şartını sağlayan durumlar için Legendre dönüşümleri mevcuttur. Bu dönüşümler hız-faz uzayı ve momentum-faz uzayı arasında bijektif dönüşümlerdir. Yozlaşmama şartını sağlamayan Lagrange ve Hamilton sistemlerinde Legendre dönüşümlerini gerçekleştirmek oldukça zor ve zahmetli bir iştir. Bu gibi durumlarda hız değişkenine karşl1ık gelen eşlenik momentum tersinir bir dönüşüm ile elde edilemeyecektir. Lagrange formalizmasından Hamilton formalizmasına geçerken Dirac-Bergmann algoritması oldukça sık başvurulan cebirsel bir yöntemdir [2-4]. Bu algoritmanın daha geometrik bir yorumu Gotay-Nester algoritması olarak bilinir [5-7].

Klasik sistemler için Lagrange fonksiyonu fiziksel sistemin hız-faz uzayında tanımlıdır. Bu tip Lagrange fonksiyonlarına birinci mertebeden Lagrange fonksiyonu diyeceğiz. İkinci bölümde ise ikinci mertebeden Lagrange fonksiyonlarını detaylı inceleyeceğiz. Birinci mertebeden Lagrange fonksiyonları en fazla ikinci dereceden diferansiyel denklem üretebilirler. Daha yüksek mertebeden diferensiyel denklemlerin Lagrange formülasyonu için bu klasik yapının dışında, örneğin ikinci mertebeden türevlere (konum-hız-ivme) bağlı bir Lagrange fonksiyonu ile başarılabilir. Yüksek mertebeden Lagrange formülasyonunun Hamilton analizi için Ostrogradski momentumları kullanılır [8,9]. Burada da momentumların hız ve ivmeden tersinir 
olarak elde edilebilmesi sorunsalı ile karşılaşılır. Tersinir olan momentumlar için Lagrange fonsiyonu Ostrogradski anlamında yozlaşmama şartını sağlar ve bu tip örnekler için yüksek mertebeden Lagrange ve Hamilton formülasyonları denktir. Fakat yozlaşmama şartını sağlamayan durumlar için Dirac-Bergmann algoritmasına başvurmak gerekecektir.

Uygun dönüşümler ile ikinci mertebeden bir Lagrange fonksiyonu birinci mertebeye indirgenebilir. Bu çalışmanın amacı öncelikle uygun dönüşümler ile ikinci mertebeden Lagrange fonksiyonlarını birinci mertebeden Lagrange fonksiyonuna indirgeme metodlarının bütüncül bir şekilde sunmaktır. Bunun için hız değişkeni yeni bir koordinat olarak tanımlanacaktır $[10,11]$. Bu tanım sayesinde iki ayrı birinci mertebe Lagrange fonksionu elde etmek mümkündür. Diğer bir indiregeme şekli ise ivme değişkenini yeni bir koordinat olarak tanımlamaktır. Bu literatürde Shmidt metodu olarak da geçmektedir [12-14]. Bu çalışmanın diğer bir ilgi alanı indirgenmiş Lagrange fonksiyonlarının Legendre dönüşümlerini başararak Hamilton analizlerini sunmaktır. Özel olarak çalışmanın ilgi alanı yozlaşmama şartını sağlamayan Lagrange fonksiyonları üzerinedir.

$\mathrm{Bu}$ hedefler doğrultusunda, çalışma 3 ana bölümden oluşturulmuştur. İlk bölümde, yozlaşmama şartını sağlamayan birinci mertebe Lagrange fonksiyonlarının Hamilton analizi özetlenmiştir. Bu bölümde Dirac-Bergmann algoritması da hatırlatılmıştır. İkinci bölümde esas amacımız olan ikinci dereceden Lagrange fonksiyonlarının indirgenmesi ve Hamilton analizleri olası 3 farklı durum için verilmiştir. Son bölümde ise yozlaşmama şartını sağlamayan ikinci mertebeden bir Lagrange teorisi olan Genel Görelilik kuramı içinde önemli bir yer tutan ve Chern-Simons terimleri ihtiva eden Chiral salınacı modeli çalışılmış, elde edilen teorik sonuçlar bu örnek üzerinde detaylı olarak incelenmiştir.

\section{BİRINCİ MERTEBEDEN LAGRANGE DİNAMIĞİ}

Bir fiziksel sistemin konfigürasyon uzayı en genel anlamda türevlenebilir katman yapısına sahiptir. Türevlenebilir katmanların boyutu yerel olarak eş yapılı olduğu öklit uzayının boyutu olarak tanımlanır. Boyutu $n$ olan $M$ katmanı yerel olarak bir koordinat sistemi $\mathbf{q}=\left(q^{1}, \ldots, q^{n}\right)$ ile donatılabilir. Fiziksel sistemin hı-faz uzayı ise katmanın tanjant demeti $T M$ 'dir. Tanjant demetleri, katmanın her noktasındaki teğet uzaylarının bir bütünüdür. Eğer katman $n$ boyutlu ise, tanjant demeti $2 n$ boyutlu bir katmanı verir. $M$ üzerindeki yerel koordinatlar aracılı̆̆ıyla $T M$ üzerinde koordinat takımı (q, $\dot{\mathbf{q}})$ ile donatılabilir. Burada q pozisyon, $\dot{\mathbf{q}}$ ise hız olarak adlandırılabilir.
Lagrange fonksiyonu $L=L(\mathbf{q}, \dot{\mathbf{q}})$ tanjant demeti $T M$ üzerinde tanımlanan reel değerli bir fonksiyondur. Etki integrali

$$
\delta=\int_{a}^{b} L(\mathbf{q}, \dot{\mathbf{q}}) d t
$$

başlangıç ve bitiş noktaları sabit olmak üzere, her farklı $\mathbf{q}=\mathbf{q}(t)$ eğrisi için farklı değerler alır. Bu integralin ekstremum değerlerini bulmak için ise integralin varyasyonu alınır ve Hamilton prensibi uygulanır. Sonuç olarak uç değer veren eğri Euler-Lagrange denklemlerini

$\frac{d}{d t} \frac{\partial L}{\partial q}-\frac{\partial L}{\partial q}=0$

sağlar. Klasik anlamda Lagrange fonksiyonu, çalışılan fiziksel sistemin kinetik ve potansiyel enerjilerinin farkı olarak yazılır. Bu özel durumda bir korunumlu sistem için Euler-Lagrange denklemleri Newton'un ikinci yasasına eşdeğerdir.

Euler-Lagrange denklemleri (2) ikinci dereceden denklemlerdir. Bu denklem takımını birinci dereceden bir denklem takımı olarak yazmak için kotanjant demeti üzerindeki Hamilton fomülasyonuna geçiş yapmak gerekir. $\mathrm{Bu}$ geçiş Legendre dönüşümü olarak adlandırılır. Bir $M$ katmanı için kotanjant demeti $T^{*} M, M$ katmanın her noktasına teğet uzayının lineer cebirsel dual uzayı $T_{x}^{*} M$ eşlenerek elde edilir. $M$ katmanı $n$ boyulu iken kotanjant demeti $T^{*} M, 2 n$ boyutlu bir katmanı verir. $T^{*} M$ üzerindeki koordinat sistemi $\mathbf{z}=(\mathbf{q}, \mathbf{p})$, pozisyonlar $\mathbf{q}$ ve momentumlar $\mathbf{p}$ olarak adlandirılabilir.

Kotanjant demetleri üzerinde kanonik simplektik iki formlar taşıllar. Simplektik 2-formlar kapalı, ters simetrik ve yozlaşmayan iki formlardır. $T^{*} M$ üzerindeki simplektik yapı $\omega_{M}$ yerel koordinat takımı $(\mathbf{q}, \mathbf{p})$ için $\omega_{M}=d \mathbf{q} \wedge d \mathbf{p}$ olarak ifade edilir. Burada $\wedge$ ile gösterilen tensör çarpımının ters simetrizasyonu ile elde edilen kama çarpımıdır. Hamilton fonksiyonu, $T^{*} M$ üzerinde tanımlı reel değerli bir fonksiyondur. Simplektik yapı $\omega_{M}$ 'nin yozlaşmama özelliği seçilen her Hamilton fonsksiyonu için $T^{*} M$ üzerinde tanımlı tek bir vektör alanı tanımlar. Bir Hamilton vektör alanı $X_{H}$ şu şekilde tanımlanır

$\iota_{X_{H}} \omega_{H}=d H$.

Burada $\iota$ büzülme operatörü, $d H$ ise Hamilton fonksiyonu $H$ 'nın dış türevidir. Hamilton vektör alanının tanımladı $\breve{g} 1$ diferansiyel denklem takımı $\dot{\mathbf{z}}=X_{H}$ (z) fiziksel sistemin hareket denklemleridir. Koordinatlar cinsinden Hamilton denklemleri

$$
\dot{\mathbf{q}}=\frac{\partial H}{\partial \mathrm{p}}, \quad \dot{\mathrm{p}}=-\frac{\partial H}{\partial \mathrm{q}}
$$


olarak elde edilir. Klasik anlamda bir Hamilton fonksiyonu fiziksel sistemin momentumlar cinsiden toplam enerjisidir. Bu durumda Hamilton denklemleri (4), Newton'un ikinci yasasına eşdeğerdir.

Simplektik iki form $\omega_{M}$, kotanjant demeti $T^{*} M$ üzerinde Poisson çerçevesi tanımlar

$\{F, H\}(\mathbf{q}, \mathbf{p})=\frac{\partial F}{\partial q} \cdot \frac{\partial H}{\partial \mathbf{p}}-\frac{\partial F}{\partial \mathbf{p}} \cdot \frac{\partial H}{\partial q} \cdot$

Poisson çerçevesi $M$ üzerindeki türevlenebilir fonksiyonlar uzayı üzerinde tanımlı ters simetrik, Leibnitz ve Jacobi özdeşliklerini sağlayan sağlayan bir işlemdir. Poisson çerçevesi cinsinden Hamilton denklemleri

\section{$\dot{\mathbf{z}}=\{\mathbf{z}, H\}$}

olarak yazılır.

Euler-Lagrange denklemleri (2) ve Hamilton denklemleri (4) arasındaki ilişki Legendre dönüşümü

$$
F L: T M \rightarrow T^{*} M:(\mathbf{q}, \dot{\mathbf{q}}) \rightarrow\left(\mathbf{q}, \frac{\partial L}{\partial \mathbf{q}}\right)
$$

aracılığıyla elde edilir. Bu dönüşüm ancak Lagrange fonksiyonu yozlaşmama şartı

$\operatorname{det}\left[\frac{\partial^{2} L}{\partial q \partial q}\right] \neq 0$

sağlandığında bire birdir. Yozlaşmama şartını sağlayan Lagrange fonksiyonlarına düzenli Lagrange fonksiyonu diyeceğiz. Bu durumda, kapalı fonksiyon teoremi gereğince, yerel olarak hız değişkenleri $\dot{\mathbf{q}}=\dot{\mathbf{q}}(\mathbf{q}, \mathbf{p})$ pozisyonlar ve momentumlar cinsinden yazılabilir. Hamilton fonksiyonu

\section{$H(\mathbf{q}, \mathbf{p})=\mathbf{p} \cdot \dot{\mathbf{q}}(\mathbf{q}, \mathbf{p})-L(\mathbf{q}, \dot{\mathbf{q}}(\mathbf{q}, \mathbf{p}))$}

olarak tanımlanır ise Hamilton denklemleri (4) Euler-Lagrange denklemleri (2)'ye dönüşür.

Düzenli olmayan, diğer bir ifade ile yozlaşmama koşulunu sağlamayan, Lagrange fonksiyonu için Lagrange ve Hamilton formalizmleri arasında bir geçiş elde elmek bu kadar kolay olamayacaktır. Legendre dönüşümü birebir ve örten olmadığından, $F L$ operatörünün görüntü uzayı kotanjant demetinin ancak bir alt katmanı olabilir ve bu durumda tüm hız değişkenleri pozisyonlar ve momentumlar cinsinden yazılamaz. Bu durumda Hamilton fonksiyonu (9) kotanjant demeti üzerinde iyi tanımlı olamayacaktır. Düzenli olmayan Lagrange fonksiyonları için Hamilton formulasyonu geçiş için bir yöntem Dirac-Bergmann algortimasıdır.

\subsection{Dirac-Bergmann Algoritması}

Legendre dönüşümü (7) birebir ve örten olmadığında hız degişsenleri $\dot{\mathbf{q}}$, pozisyonlar $\mathbf{q}$ ve momentumlar $\mathbf{P}$ cinsinden yazılamaz ve fakat
$\Phi_{m}(\mathbf{q}, \mathbf{p}) \simeq 0, \quad m=1, \ldots, M$

şeklinde bağıntılar verir. Bu bağıntılar birincil kısıtlar olarak adlandırılır ve kotanjant demetinin bir alt uzayını oluşstururlar [2, 3]. Bu alt uzayda tüm (bütül) Hamilton fonksiyonu birincil kisıtların eklenmesiyle elde edilir

$$
H_{T}=H+\mathrm{U}^{m} \Phi_{m}
$$

Burada $\mathbf{U}^{m}$ (q, p) katsavıları Lagrange çarpanları olarak adlandırılır. Bu durumda, ${ }_{T}$ için Hamilton denklemleri (4)

$\dot{\mathbf{q}}=\frac{\partial H}{\partial \mathrm{p}}+U^{m} \frac{\partial \phi_{m}}{\partial \mathrm{p}}, \dot{\mathbf{p}}=-\frac{\partial H}{\partial \mathrm{q}}-U^{m} \frac{\partial \phi_{m}}{\partial \mathrm{q}}$

denklemlerine dönüşür. Diğer taraftan (6)' da tanımlanan Hamilton denklemlerinde birincil kısıt $\Phi_{m}$ foksiyonları yerleştirilirse $m=1, \ldots, M$ için

$\Phi_{m}=\left\{\Phi_{m}, H\right\}+U^{n}\left\{\Phi_{n}, \Phi_{m}\right\} \simeq 0$

denklemleri elde edilir, bu denklemlere tutarlılık koşulları denir. Bu denklemlerde eğer $\operatorname{det}\left\{\Phi_{n}, \Phi_{m}\right\} \neq 0$ ise, Lagrange çarpanları $U^{n}$ belirlenebilir. Fakat $\operatorname{det}\left\{\Phi_{n}, \Phi_{m}\right\} \simeq 0$ ise tutarlılık koşulları $U^{n}$, li terim içermez ve ikincil kısıt olarak adlandırılan $\chi(\mathbf{q}, \mathbf{p}) \simeq 0$ ifadeleri elde edilir. İkincil kısıtlar için de tutarlılık koşulları kontrol edilmeli, mümkünse Lagrange çarpanları belirlenmeli, değilse elde edilen yeni kısıtlar (üçüncül, dördüncül v.b.) için tutarlılık koşullarına bakılmalıdır. Bu süreç cebir kapanan kadar devam ettirilmelidir. Cebir kapandığında, elde edilen tüm kısıtlar, kotanjant demetinin bir alt katmanını, son kisıt katmanını, belirler. Bu altkatmanda Hamilton denklemleri tutarlıdır ve hareket denklerimini üretirler.

\section{III. İkinci Mertebeden Lagrange Dinamiği ve İndirgemeleri}

Bir $n$ boyutlu $M$ katmanı için tanjant demeti $T M$ pozisyon (q) ve hızlardan ( $\dot{\mathbf{q}}$ ) müteşekkildir ve $2 n$ boyutludur. İkinci mertebeden tanjant demeti $T^{2} M$ ise pozisyon (q), hiz ( $\left.\dot{\mathbf{q}}\right)$ ve ivmeden ( $\mathbf{q})$ oluşur, ve $3 n$ boyutludur.

Tanjant demeti $T M$ tek başına bir katman yapısına sahip olduğundan onun da teğet demeti $T(T M)=T T M$ demeti tanımlanabilir. Burada, $M$ katmanı $n$ boyutlu ise, $T M$ katmanı $2 n, T T M$ is $4 n$ boyutludur. İkinci mertebeden tanjant demeti $T^{2} M$, ikinci tanjant demeti $T T M$ içine doğal bir yataklanmaya sahiptir. Yerel koordinatlarda bu yataklanma

$$
\begin{aligned}
& T^{2} M \rightarrow T T M:(\mathbf{q}, \dot{\mathbf{q}}, \ddot{\mathbf{q}}) \rightarrow(\mathbf{q}, \dot{\mathbf{q}} ; \dot{\mathbf{q}}, \overrightarrow{\mathbf{q}}) \\
& \quad \text { olarak ifade edilir. }
\end{aligned}
$$

İkinci mertebeden bir Lagrange fonksiyonu $L=L(\mathbf{q}, \dot{\mathbf{q}}, \mathbf{q})$ ikinci mertebeden tanjant demeti $T^{2} M$ 
üzerinde tanımlı reel değerli bir fonksiyondur. Bu fonksiyon için de etki integrali tanımlanabilir:

$\mathcal{S}=\int_{a}^{b} L(\mathbf{q}, \dot{\mathbf{q}}, \ddot{\mathbf{q}}) d t$.

$\mathrm{Bu}$ integral her $\mathbf{q}=\mathbf{q}(t)$ için farklı değerler alacaktır. Etki integralinin varyasyonunu

$\delta \delta=\int_{a}^{b}\left(\frac{\partial L}{\partial \mathrm{q}}-\frac{d}{d t} \frac{\partial L}{\partial \mathrm{q}}+\frac{d^{2}}{d t^{2}} \frac{\partial L}{\partial \mathrm{q}}\right) \cdot \delta \mathrm{q} d t$

şeklindedir. Burada $\delta \mathbf{q}$ rasgele bir teğet vektörü olarak düşünülürse, varyasyonun sıfır değerini alması, diğer bir ifade ile etki integralinin uç değere ulaşması için gerek koşul ikinci mertebeden Euler-Lagrange denklemlerinin

$\frac{d^{2}}{d t^{2}} \frac{\partial L}{\partial q}-\frac{d}{d t} \frac{\partial L}{\partial q}+\frac{\partial L}{\partial q}=0$

sağlanması olarak bulunur. Burada dikkat edilmesi gereken ikinci mertebeden Euler-Lagrange denklemleri (17)'nin eğer $\partial L / \partial$ q̈ açık olarak q̈'ya bağlıysa dördüncü mertebeden diferansiyel denklem takımı, eğer $\partial L / \partial$ q̈ açık olarak q̈ 'a bağlı değil ve fakat q̇'ya bağlıysa üçüncü mertebeden diferansiyel denklem takımı üretmesidir.

\subsection{Birinci Mertebeye İndirgeme}

\subsubsection{Mertebe indirgeme I}

$2 n$ boyutlu konfigürasyon uzayı $N=T M$ 'nın koordinatları

$$
\mathbf{q}_{(1)}=\mathbf{q}, \quad \mathbf{q}_{(2)}=\dot{q} \text {. }
$$

olarak yeniden adlandırılabilir ve $\dot{\mathbf{q}}_{(1)}-\mathbf{q}_{(2)}=0$ k1sitlardir.

$T\left(T M \times \mathbb{R}^{n}\right)$ uzayında Lagrange fonksiyonu ise şu şekilde tanımlanır:

$L_{C_{1}}=L\left(\mathbf{q}_{(1)}, \mathbf{q}_{(2)}, \dot{\mathbf{q}}_{(2)}\right)+\lambda_{1} \cdot\left(\dot{\mathbf{q}}_{(1)}-\mathbf{q}_{(2)}\right)$

burada $\lambda_{1}$ Lagrange çarpanıdır ve $\mathbf{q}_{(1)}, \mathbf{q}_{(2)}, \dot{\mathbf{q}}(1)$ ve $\dot{\mathbf{q}}_{(2)}$ 'in fonksiyonudur. (2) de tanımlanan birinci mertebeden Euler-Lagrange denklemleri $L_{c_{1}}$ için

$$
\begin{aligned}
& \frac{\partial L_{C_{1}}}{\partial \mathbf{q}_{(1)}}-\lambda_{1}=0, \quad \frac{\partial L_{C_{1}}}{\partial \mathbf{q}_{(2)}}-\frac{d}{d t} \frac{\partial L_{C_{1}}}{\partial \dot{\mathbf{q}}_{(2)}}=\lambda_{1}, \\
& \dot{\mathbf{q}}_{(1)}-\mathbf{q}_{(2)}=0
\end{aligned}
$$

olarak yazılabilir. (20) denklemleri ikinci dereceden Euler-Lagrange denklemleri (17)’ ye denktir.

Kotanjant demeti $T^{*}\left(T Q \times \mathbb{R}^{n}\right)$ de eşlenik momentumlar

$$
\mathbf{p}^{(1)}=\frac{\partial L}{\partial q_{(1)}}, \mathbf{p}^{(2)}=\frac{\partial L}{\partial q_{(2)}}, \mathbf{p}^{\lambda}=\frac{\partial L}{\partial \lambda_{1}}
$$

şeklinde tanımlanır. Bu durumda momentumların tanımindan birincil kisitlar

$\Phi^{(1)}=\mathbf{p}^{(1)}-\lambda_{1} \simeq 0$,

$\Phi^{(2)}=\mathbf{p}^{(2)}-\frac{\partial L}{\partial q_{2}} \simeq 0$,

$\Phi^{\left(\lambda_{1}\right)}=\mathbf{p}^{\lambda_{1}} \simeq 0$

olarak elde edilir. Hamilton fonksiyonu

$H_{C_{1}}=\mathbf{p}^{(i)} \cdot \dot{\mathbf{q}}_{(\mathrm{i})}+\lambda_{1} \cdot \mathbf{p}^{\lambda_{1}}-L_{C_{1}}=\mathbf{p}^{(\mathrm{i})} \cdot \dot{\mathbf{q}}_{(\mathrm{i})}-L,(22)$

olur. Burada $i$ indisi 1 ve 2 değerlerini alır, ve toplama uylaşımı kabul edilmiştir. Toplam Hamilton fonksiyonu (21)'de tanımlanan birincil kisitları Hamilton fonksiyonuna eklerek tanımlanır:

$H_{\tau_{1}}=H_{C_{1}}+\mathbf{u}_{(\alpha)} \cdot \Phi^{(\alpha)}$

burada $\alpha$ indisi $\left\{1,2, \lambda_{1}\right\}$ değerlerini alır ve $\mathbf{u}_{(\alpha)}$ lar Lagrange çarpanlarıdır. Birincil kısıtlar için (13)'de tanımlanan tutarlılık koşulları

$\left\{H_{\tau_{1}}, \Phi^{(1)}\right\}=-\frac{\partial H}{\partial \mathbf{q}_{(1)}}-\mathbf{u}_{\left(\lambda_{1}\right)} \simeq 0$,

$\left\{H_{\tau_{1}}, \Phi^{\left(\lambda_{1}\right)}\right\}=\mathbf{u}_{(1)} \simeq 0$

denklemlerini verir. Bu denklemlerden $\mathbf{u}_{\left(\lambda_{1}\right)}$ ve $\mathbf{u}_{(1)}$ belirlenebilir fakat $\mathbf{u}_{(2)}$ ' yi belirlemek için $\Phi^{(2)}$ k1sıtının tutarlılık koşulu incelenmelidir.

\subsubsection{Mertebe indirgeme II}

Bölüm 3.1.1 de verilen birinci mertebeye indirgemeye ek olarak ḋ kordinatı $\dot{q}_{(1)}$ gibi düşünülerek farklı bir Lagrange fonksiyonu

$L_{C_{2}}=L\left(\mathbf{q}_{(1)}, \dot{\mathbf{q}}_{(1)}, \dot{\mathbf{q}}_{(2)}\right)+\lambda_{2} \cdot\left(\dot{\mathbf{q}}_{(1)}-\mathbf{q}_{(2)}\right)$,

elde edilebilir. Burada $\lambda_{2}$ başka bir Lagrange çarpan1dır. $L_{C_{2}}$ tarafindan üretilen Euler-Lagrange denklemleri ise şu şekildedir:

$\frac{\partial L_{C_{2}}}{\partial \mathbf{q}_{(1)}}-\frac{d}{d t} \frac{\partial L_{C_{2}}}{\partial \dot{\mathbf{q}}_{(1)}}-\lambda_{2}=0, \quad-\frac{d}{d t} \frac{\partial L_{C_{2}}}{\partial \dot{\mathbf{q}}_{(2)}}=\lambda_{2}$,

$\dot{\mathbf{q}}_{(1)}-\mathbf{q}_{(2)}=0$.

$\mathrm{Bu}$ hareket denklemleri de (17)'deki ikinci mertebeden Euler-Lagrange denklemlerine denktir.

\subsubsection{Schmidt metodu ile birinci mertebeye indirgeme}

Bölüm (3.1.1) ve (3.1.2)'de bahsi geçen mertebe indirgemelerinden farklı olarak 


$$
\mathbf{q}_{(1)}=\mathbf{q}, \quad \mathbf{q}_{(3)}=\ddot{\mathbf{q}},
$$

yerel kordinat dönüşümleri ile, TTM ' nin $2 n$ boyutlu alt katmanı

$$
A M=\left\{X \in T T M: \tau_{T M}(X)=T \tau_{M}(X)=0\right\}
$$

şeklinde tanımlanır ve $A M$ için yerel harita ikilileri $\left(\mathbf{q}_{(1)}, \mathbf{q}_{(3)}\right)$ olur. $A M$ ' nun teğet uzayı $T A M$ de indirgenmiş koordinatlar

$\left(\mathbf{q}_{(1)}, \mathbf{q}_{(3)} ; \dot{\mathbf{q}}_{(1)}, \dot{\mathbf{q}}_{(3)}\right) \in T A M$

şeklindendir, ve

\section{$\theta: T^{3} M \mapsto T A M:(\mathbf{q} ; \dot{\mathbf{q}} ; \mathbf{q} ; \ddot{\mathbf{q}}) \mapsto(\mathbf{q} ; \ddot{\mathbf{q}} ; \dot{\mathbf{q}} ; \ddot{\mathbf{q}})$}

yerel tanımlama ile $T A M$ teğet uzayı $T^{3} M$ uzayına izomofiktir.

İkinci derece bir ${ }^{L}$ Lagrange fonksiyonunu ele alalım. İvme kordinat1 $\mathbf{q}_{(3)}$ ' $\ddot{\mathbf{u}} \dot{\mathbf{q}}_{(1)}$ '1n türevi gibi düşünmek için, $T A M$ teğet demeti üzerinde $T A M \times T N$ yapısı inşa edilir. Burada $N$ yerel kordinatları (r) olan $n$ - boyutlu katmandır. (29) de verilen izomorfizma ile $T A M \times T N$ de, birinci mertebeden Lagrange fonksiyonu tanımlanabilir:

$$
\begin{aligned}
& L_{3}\left(\mathbf{q}_{(1)}, \mathbf{q}_{(3)}, \dot{\mathbf{q}}_{(1)}, \dot{\mathbf{q}}_{(3)}, \mathbf{r}, \dot{\mathbf{r}}\right)=L\left(\mathbf{q}_{(1)}, \dot{\mathbf{q}}_{(1)}, \mathbf{q}_{(3)}\right)+ \\
& \frac{\partial F}{\partial \mathbf{q}_{(1)}} \cdot \dot{\mathbf{q}}_{(1)}+\frac{\partial F}{\partial \mathbf{q}_{(1)}} \cdot \mathbf{q}_{(3)}+\frac{\partial F}{\partial \mathbf{q}_{(3)}} \cdot \dot{\mathbf{q}}_{(3)}+\frac{\partial F}{\partial \mathbf{r}} \cdot \dot{\mathbf{r}} .
\end{aligned}
$$

Burada $F$ fonksiyonu $\left(\mathbf{q}_{(1)}, \mathbf{q}_{(3)}, \dot{\mathbf{q}}_{(1)}, \mathbf{r}\right)$ koordinatlarına bağımlı olarak secilir. Kolaylıkla görülecektir ki, $L_{3}$ tarafindan üretilen Euler-Lagrange denklemleri (17)'de verilen ikinci derece Euler-Lagrange denklemlerine denktir. Bu $\operatorname{denklik}\left[\partial^{2} F / \partial \dot{\mathbf{q}}_{(1)} \partial \mathbf{r}\right]$ matrisinin dejenere olmaması durumunda geçerlidir. En basit şekilde $F=\dot{\mathbf{q}}_{(1)} \cdot \mathbf{r}$ şeklinde al1nabilir ve bu durumda Lagrange fonksiyonu

$$
\begin{aligned}
& L_{3}\left(\mathbf{q}_{(1)}, \mathbf{q}_{(3)}, \dot{\mathbf{q}}_{(1)}, \dot{\mathbf{q}}_{(3)}, \mathbf{r}, \dot{\mathbf{r}}\right)=L\left(\mathbf{q}_{(1)}, \dot{\mathbf{q}}_{(1)}, \mathbf{q}_{(3)}\right)+ \\
& \mathbf{r} \cdot \mathbf{q}_{(3)}+\dot{\mathbf{q}}_{(1)} \cdot \dot{\mathbf{r}}
\end{aligned}
$$

şeklinde yazılabilir.

$T^{*}(A M \times N)$ de eşlenik momentum kordinatları $\left(\mathbf{p}^{(1)}, \mathbf{p}^{(3)}, \mathbf{p}^{(r)}\right)$ dir, ve söyle hesaplanır:

$$
\mathbf{p}^{(1)}=\frac{\partial L}{\partial \dot{\mathbf{q}}_{(1)}}\left(\mathbf{q}_{(1)}, \dot{\mathbf{q}}_{(1)}, \mathbf{q}_{(3)}\right)+\dot{\mathbf{r}}
$$

$\mathbf{p}^{(3)}=\mathbf{0}, \quad \mathbf{p}^{(r)}=\dot{\mathbf{q}}^{(1)} \mathbf{p}^{(3)}=\mathbf{0}, \quad \mathbf{p}^{(r)}=\dot{\mathbf{q}}^{(1)}$.

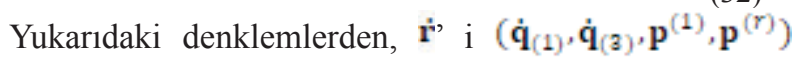
cinsinden çözebiliriz. Fakat $\varphi_{1}=\mathbf{p}^{(3)} \simeq 0$ birincil kısıttır. Toplam Hamilton fonksiyonu

$$
H_{\tau}=\mathbf{p}^{(1)} \cdot \mathbf{p}^{(r)}-L\left(\mathbf{q}_{(1)}, \mathbf{p}^{(r)}, \mathbf{q}_{(3)}\right)-\mathbf{r} \cdot \mathbf{q}_{(3)}+\lambda \cdot
$$

şeklindedir ve birincil kısıtın korunması

$$
\varphi_{2}=\left\{\varphi_{1}, H_{T}\right\}=\left\{\mathbf{p}^{(3)}, H_{\tau}\right\}=\frac{\partial L}{\partial \mathbf{q}_{(3)}}+\mathbf{r}=\mathbf{0} .
$$

yeni bir kısıt $\varphi_{2}$ 'yi verir. Benzer şekilde, ikincil kısıt olarak adlandırılan bu kısıtın korunmasından üçüncül kısıt

$$
\begin{aligned}
& \varphi_{3}=\left\{\varphi_{2}, H_{T}\right\}=\left(\lambda \cdot \frac{\partial}{\partial \mathbf{q}_{(3)}}+\mathbf{q}_{(3)} \cdot \frac{\partial}{\partial \mathbf{p}^{(r)}}+\mathbf{p}^{(r)} .\right. \\
& \left.\frac{\partial}{\partial \mathbf{q}_{(1)}}\right)\left(\frac{\partial L}{\partial \mathbf{q}_{(3)}}\right)+\mathbf{p}^{(1)}=0
\end{aligned}
$$

elde edilir. Eğer Lagrange fonksiyonunun yozlaşmamış olduğu kabul edilirse bu noktada Lagrange çarpanı $\lambda$ belirlenir ve algoritma sonlanır. Fakat Lagrange fonksiyonu yozlaşmış ise Lagrange çarpanını belirlemek için bir kaç adım daha devam etmek gerekebilir [4].

\section{CHIRAL SALINAÇI}

İki boyutlu Chern-Simons terimlerini içeren, Chiral salınacı aşağıda verilen Lagrange fonksiyonu tarafindan tanımlanır:

$L\left(x^{i}, \dot{x}^{i}, \ddot{x}^{i}\right)=-\frac{\lambda}{2} \epsilon_{i j} \dot{x}^{i} \ddot{x}^{j}+\frac{m}{2} \dot{x}^{i} \dot{x}_{i}$.

Burada $\lambda$ ve $m$ sifirdan farklı sabitler, $x^{i}=(x, y)$ ve $\epsilon_{i j}$ Levi-Civita sembolüdür, $[15,16]$.

Chiral Lagrange fonksiyou için (17)' de verilen Euler-Lagrange denklemleri

$\lambda \epsilon_{i j} \ddot{x}^{j}-m \ddot{x}_{i}=0$

olarak elde edilir. Bu denklemler 3. Mertebeden olup, bunun sebebi (36)' de verilen Chiral Lagrange fonksiyonunun yozlaşmış olmasıdır. Diğer bir ifade ile yozlaşmama şartı

$$
\begin{aligned}
& \operatorname{det}\left[\frac{\partial^{2} L}{\partial \ddot{x}^{i} \partial \ddot{x}^{j}}\right]=0 \\
& \quad \text { denkleminin sağlanmamasıdır. }
\end{aligned}
$$

\subsection{Mertebe İndirgeme I}

Chiral salınaçı için (19)'da tanımlanan birinci mertebeye indirgenmiş Lagrangian

$$
\begin{aligned}
& L_{C_{1}}=-\frac{\lambda}{2} \epsilon_{i j} q_{(2)}^{i} \dot{q}_{(2)}^{j}+\frac{m}{2} \delta_{i j} q_{(2)}^{i} q_{(2)}^{j}+\beta_{i}\left(\dot{q}_{(1)}^{i}-\right. \\
& \left.q_{(2)}^{i}\right)
\end{aligned}
$$

olur ve burada $\beta_{i}$ Lagrange çarpanıdır. $L_{C_{1}}$ için eşlenik momentumlar

$$
p_{i}^{(1)}=\beta_{i}, \quad p_{i}^{(2)}=-\frac{\lambda}{2} \epsilon_{j i} q_{(2)}^{j}, \quad p_{\beta}^{i}=0
$$

şeklindedir. Bu momentumlardan $\dot{q}_{(1)}^{i}, \dot{q}_{(2)}^{i}$ ve $\beta_{j}$ 'leri çözmek mümkün olmadığı için (21) denklemindeki gibi tanımlanan birincil kısitlar 


$$
\begin{aligned}
& \Phi_{i}^{(1)}=p_{i}^{(1)}-\beta_{i}, \\
& \Phi_{i}^{(2)}=p_{i}^{(2)}+\frac{\lambda}{2} \epsilon_{j i} q_{(2)}^{j}, \\
& \Phi_{\beta}^{i}=p_{\beta}^{i}
\end{aligned}
$$

olarak elde edilir. (22) denkleminden Hamilton fonksiyonu

$$
H=-\frac{m}{2} \delta_{i k} q_{(2)}^{i} q_{(2)}^{k}+\beta_{i} q_{(2)}^{i}
$$

ve (23) denkleminden de toplam Hamilton fonksiyonu şu şekilde tanımlanır:

$$
H_{T}=H+\Phi_{i}^{(1)} u_{(1)}^{i}+\Phi_{i}^{(2)} u_{(2)}^{i}+\Phi_{\beta}^{i} u_{i}^{\beta} .
$$

Burada $u_{(1)}^{i}, u_{(2)}^{i}$ ve $u_{i}^{\beta}$ Lagrange çarpanlarıdır. Birincil kısıtlar için (13)'de tanımlanan tutarlılık koşullarından

$$
\begin{aligned}
& \Phi_{i}^{(1)}=\left\{\Phi_{i}^{(1)}, H_{T}\right\}=-u_{i}^{\beta}, \\
& \Phi_{i}^{(2)}=\left\{\Phi_{i}^{(2)}, H_{T}\right\}=m \delta_{i k} q_{(2)}^{k}-\beta_{i}-\lambda \epsilon_{i j} u_{(2)}^{j}, \\
& \Phi_{\beta}^{i}=\left\{\Phi_{\beta}^{i}, H_{T}\right\}=-q_{(2)}^{i}+u_{(1)}^{i},
\end{aligned}
$$

Lagrange çarpanları

$$
u_{i}^{\beta}=0, \quad u_{(1)}^{i}=q_{(2)}^{i}, \quad u_{(2)}^{i}=\frac{\epsilon^{i j}\left(-m \delta_{j k} q_{(2)}^{k}+\beta_{j}\right)}{\lambda}
$$

olarak belirlenir. Bunların toplam Hamilton fonksiyonu (42)

$H_{T}=-\frac{1}{2} \beta_{i} q_{(2)}^{i}+p_{i}^{(1)} q_{(2)}^{i}+\frac{1}{\lambda} e^{i k} p_{i}^{(2)}\left(-m \delta_{k l} q_{(2)}^{l}+\right.$

$$
\left.\beta_{k}\right)
$$

olarak elde edilir. Hamilton denklemleri ise şu şekilde olur:

$$
\begin{aligned}
& \dot{q}_{(1)}^{i}=\left\{q_{(1)}^{i}, H_{T}\right\}=q_{(2)}^{i}, \\
& \dot{q}_{(2)}^{i}=\left\{q_{(2)}^{i}, H_{\tau}\right\}=\frac{1}{\lambda} \epsilon^{i k}\left(-m \delta_{k l} q_{(2)}^{l}+\beta_{k}\right), \\
& \beta_{i}=\left\{\beta_{i}, H_{\tau}\right\}=0, \\
& \dot{p}_{i}^{(1)}=\left\{p_{i}^{(1)}, H_{T}\right\}=0, \\
& \dot{p}_{i}^{(2)}=\left\{p_{i}^{(2)}, H_{T}\right\}=-p_{i}^{(1)}+\frac{m}{\lambda} \delta_{i k} e^{j k} p_{j}^{(2)}+\frac{1}{2} \beta_{i}, \\
& \dot{p}_{\beta}^{i}=\left\{p_{\beta}^{i}, H_{\tau}\right\}=-\frac{1}{\lambda} \epsilon^{j i} p_{j}^{(2)}+\frac{1}{2} q_{(2)}^{i} .
\end{aligned}
$$

Bu hareket denklemlerden $\beta_{i}$ ve $\dot{p}_{i}^{(1)}$, de momentum (49) de verilen tanımları yazılırsa Euler-Lagrange denklemleri
(37) elde edilir. Diğer hareket denklemleri ise özdeş olarak sağlanır.

\subsection{Mertebe İndirgeme II}

Benzer şekilde, (25)'de tanımlanan birinci mertebeden alternatif Lagrangian, Chiral Lagrange fonksiyonunu (36) için yazılırsa

$$
\begin{aligned}
& L_{c_{2}}=-\frac{\lambda}{2} \epsilon_{i j} \dot{q}_{(1)}^{\mathrm{i}} \dot{q}_{(2)}^{j}+\frac{m}{2} \delta_{i j} \dot{q}_{(1)}^{\mathrm{i}} \dot{q}_{(1)}^{j}+\alpha_{i}\left(\dot{q}_{(1)}^{\mathrm{i}}-\right. \\
& \left.q_{(2)}^{\mathrm{i}}\right)
\end{aligned}
$$

elde edilir, $\alpha_{i}$ Lagrange çarpanıdır. $L_{c_{2}}$ için eşlenik momentumlar şu şekildedir:

$r_{i}^{(1)}=-\frac{\lambda}{2} \epsilon_{i j} \dot{q}_{(2)}^{j}+m \delta_{i j} \dot{q}_{(1)}^{j}+\alpha_{i}$,

$r_{i}^{(2)}=-\frac{\lambda}{2} \epsilon_{j i} \dot{q}_{(1)}^{j}$,

$r_{\alpha}^{i}=0$.

Bu momentumlardan $\dot{q}_{(1)}^{i}, \dot{q}_{(2)}^{i}$ çözülebilir:

$\dot{q}_{(1)}^{i}=-\frac{2}{\lambda} e^{i j} r_{j}^{(2)}$,

$\dot{q}_{(2)}^{i}=-\frac{2}{\lambda} \epsilon^{i j}\left(\alpha_{j}-r_{j}^{(1)}\right)-\frac{4 m}{\lambda^{2}} \delta^{i j} r_{j}^{(2)}$

fakat $\dot{\alpha}_{j}$ 'ler çözülemez ve birincil kısıtlar $\Phi_{\alpha}^{i}=r_{\alpha}^{i}$ elde edilir. Hamilton fonksiyonu

$$
\begin{aligned}
& H=-\frac{2}{\lambda} \epsilon^{j k} r_{j}^{(1)} r_{k}^{(2)}-\frac{2 m}{\lambda^{2}} \delta^{j k} r_{j}^{(2)} r_{k}^{(2)}+\frac{2}{\lambda} \epsilon^{j k} \alpha_{j} r_{k}^{(2)}+ \\
& \alpha_{j} q_{(2)}^{j}
\end{aligned}
$$

ve toplam Hamilton fonksiyonu şu şekilde tanımlanır:

$$
H_{\tau}=H+\Phi_{\alpha}^{i} u_{i}^{\alpha}
$$

burada $u_{i}^{\alpha}$ Lagrange çarpanlarıdır. Birincil kısıtların korunmasindan

$$
\Phi_{\alpha}^{i}=\left\{\Phi_{\alpha}^{i}, H_{\tau}\right\}=-\frac{2}{\lambda} e^{i k} r_{k}^{(2)}-q_{(2)}^{i}
$$

ikincil kısıt $\Phi^{i}=\frac{2}{\lambda} e^{i k} r_{k}^{(2)}-q_{(2)}^{i}$ elde edilir. Bu ikincil kisitın korunmasindan

$$
\Phi^{i}=\left\{\Phi^{i}, H_{\tau}\right\}=-\frac{4}{\lambda} \epsilon^{i k} \alpha_{k}-\frac{2}{\lambda} e^{j i} r_{j}^{(1)}-\frac{4 m}{\lambda^{2}} \delta^{i j} r_{j}^{(2)}
$$

yeni bir kısit elde edilir

$$
\phi=2 \epsilon^{i k} \alpha_{k}+\epsilon^{j i} r_{j}^{(1)}+\frac{2 m}{\lambda} \delta^{i j} r_{j}^{(2)} .
$$

Son olarak bu kisitın da korunmasından 


$$
\dot{\phi}^{i}=\left\{\phi^{i}, H_{\tau}\right\}=2 \epsilon^{i k} u_{k}^{\alpha}-\frac{2 m}{\lambda} \delta^{i j} \alpha_{j}
$$

Lagrange çarpanı $u_{i}^{\alpha}=-\frac{m}{\lambda} \epsilon_{i j} \delta^{j k} \alpha_{k}$ belirlenir. Belirlenen $u_{i}^{\alpha}$ ların toplam Hamilton fonksiyonu ${ }^{(52)}$ de yazılmasiyla

$H_{T}=-\frac{2}{\lambda} \epsilon^{j k} r_{j}^{(1)} r_{k}^{(2)}-\frac{2 m}{\lambda^{2}} \delta^{j k} r_{j}^{(2)} r_{k}^{(2)}+$

$\frac{2}{\lambda} \epsilon^{j k} \alpha_{j} r_{k}^{(2)}+\alpha_{j} q_{(2)}^{j}-\frac{m}{\lambda} \epsilon_{j i} \delta^{i k} r_{\alpha}^{j} \alpha_{k}$

elde edilir. Toplam Hamilton fonksiyonu (56)'yi kullanarak, Hamilton hareket denklemleri şu şekilde elde edilir:

$$
\begin{aligned}
& \dot{q}_{(1)}^{i}=-\frac{2}{\lambda} \epsilon^{i k} r_{k}^{(2)}, \\
& \dot{q}_{(2)}^{i}=-\frac{2}{\lambda} e^{j i} r_{j}^{(1)}-\frac{4 m}{\lambda^{2}} \delta^{i k} r_{k}^{(2)}+\frac{2}{\lambda} \epsilon^{j i} \alpha_{j}, \\
& \dot{\alpha}_{i}=-\frac{m}{\lambda} \epsilon^{i j} \alpha_{j}, \\
& \dot{p}_{i}^{(1)}=0, \\
& \dot{p}_{i}^{(2)}=-\alpha_{i}, \\
& \dot{p}_{\alpha}^{i}=-\frac{2}{\lambda} e^{i k} r_{k}^{(2)}-q_{(2)}^{i} .
\end{aligned}
$$

Bu hareket denklemlerinden $\dot{\alpha}_{i}$ ve $\dot{r}_{i}^{(1)}$ de momentumların tanımları yazılırsa Euler-Lagrange denklemleri (37) elde edilir. Diğer denklemler özdeş olarak sağlanır.

\subsection{Chiral Lagrange için Schmidt metodu}

Chiral salınaç için Lagrange fonksiyonu (3.1.3)'de belirtilen yöntem kullanılarak birinci mertebeye indirgenebilir. Bunun için $\ddot{x}^{i}=s^{i}, r^{i}=\frac{\partial L}{\partial s^{i}}=\frac{\lambda}{2} \epsilon_{i j} \dot{x}^{j}$ koordinat dönüşümleri ile (31)' de tanımlanan Lagrange fonksiyonu

$$
L_{3}=\frac{m}{2} \delta_{i j} \dot{x}^{i} \dot{x}^{j}-\frac{\lambda}{2} \epsilon_{i j} \dot{x}^{i} s^{j}+\delta_{i j} \dot{r}^{i} \dot{x}^{j}+\delta_{i j} r^{i} s^{j}
$$

şeklinde yazılabilir. Bu durumda (32)'de tanımlanan eşlenik momentum koordinatları

$$
\begin{aligned}
& p_{i}^{x}=m \delta_{i j} \dot{x}^{j}-\frac{\lambda}{2} \epsilon_{i j} s^{j}+\delta_{i j} \dot{r}^{j}, \\
& p_{i}^{r}=\delta_{i j} \dot{x}^{j}, \\
& p^{s}=0
\end{aligned}
$$

olarak hesaplanır. Bu momentum tanımlarından $\dot{x}^{i} \mathrm{ve}^{\dot{r}^{j}}$ aşağıdaki şekilde çözülebilir:

$$
\dot{x}^{i}=\delta^{i j} p_{j}^{r}, \dot{r}^{i}=\delta^{i j}\left(p_{j}^{x}-m p_{j}^{r}\right)+\frac{\lambda}{2} \delta^{i k} \epsilon_{k j} s^{j},
$$

ve diğer momentum $\Phi_{i}^{s}=p_{i}^{s}=0$ birincil kısitlayıcıdır. (33)'de tanımlanan toplam Hamilton fonksiyonu

$$
\begin{aligned}
& H_{\tau}=\delta^{i j} p_{i}^{x} p_{j}^{r}-\frac{m}{2} \delta^{i j} p_{i}^{r} p_{j}^{r}+\frac{\lambda}{2} \epsilon_{i j} \delta^{i k} p_{k}^{r} s^{j}- \\
& \delta_{i j} r^{i} s^{j}+u^{i} p_{i}^{s}
\end{aligned}
$$

şeklindedir ve burada $u^{i}$ Lagrange çarpanıdır. Birincil kisitın korunmasindan

$$
\Phi_{i}^{s}=p_{i}^{s}=\left\{p_{i}^{s}, H_{\tau}\right\}=-\frac{\lambda}{2} \epsilon_{j i} \delta^{j k} p_{k}^{r}+\delta_{j i} r^{j}
$$

ikincil kısit

$$
\phi_{i}=-\frac{\lambda}{2} \epsilon_{j i} \delta^{j k} p_{k}^{r}+\delta_{j i} r^{j}
$$

elde edilir. Bu kısıtında korunmasından ise

$$
\begin{gathered}
\dot{\phi}_{i}=\left\{\phi_{i}=, H_{\tau}\right\}=\lambda \epsilon_{i j} s^{j}+p_{i}^{x}-m p_{i}^{r} \\
\text { üçüncül kisıt }
\end{gathered}
$$

$$
\alpha_{i}=\lambda \epsilon_{i j} s^{j}+p_{i}^{x}-m p_{i}^{r}
$$

$$
\text { gelir ve son olarak üçüncül kısıtın korunmasından }
$$

$$
\dot{\alpha}_{i}=\left\{\alpha_{i}, H_{\tau}\right\}=\lambda \epsilon_{i j} u^{j}-m \delta_{i j} s^{j}=0
$$

\section{Lagrange çarpanı}

$u^{i}=\frac{-m}{\lambda} \epsilon^{i j} \delta_{j k} s^{k}$

olarak belirlenir ve bu $u^{i}$ ', toplam Hamilton fonksiyonu (62)' de yazılmasıyla toplam Hamilton fonksiyonu aşağıdakı gibi elde edilir:

$$
\begin{aligned}
& H_{\tau}=\delta^{i j} p_{i}^{x} p_{j}^{r}-\frac{m}{2} \delta^{i j} p_{i}^{r} p_{j}^{r}+\frac{\lambda}{2} \epsilon_{i j} \delta^{i k} p_{k}^{r} s^{j}- \\
& \delta_{i j} r^{i} s^{j}-\frac{m}{\lambda} \epsilon^{i j} \delta_{j k} s^{k} p_{i}^{s} .
\end{aligned}
$$

Hamilton fonksiyonu (66)'yı kullanarak Hamilton denklemleri

$$
\begin{aligned}
& \dot{x}^{i}=\left\{x^{i}, H_{T}\right\}=\delta^{i j} p_{j}^{r} \dot{x}^{i}=\left\{x^{i}, H_{T}\right\}=\delta^{i j} p_{j}^{r} \\
& \dot{r}^{i}=\left\{r^{i}, H_{T}\right\}=\delta^{i j} p_{j}^{x}-m \delta^{i j} p_{j}^{r}+\frac{\lambda}{2} \epsilon_{i j} s^{j} \\
& \dot{s}^{i}=\left\{s^{i}, H_{T}\right\}=-\frac{m}{\lambda} \delta^{i k} \epsilon_{k j} s^{j} \\
& \dot{p}^{x}{ }_{i}=\left\{p_{i}^{x}, H_{\tau}\right\}=0 \dot{p}^{x}{ }_{i}=\left\{p_{i}^{x}, H_{T}\right\}=0 \\
& \dot{p}^{r}{ }_{i}=\left\{p_{i}^{r}, H_{\tau}\right\}=\delta_{i j} s^{j}
\end{aligned}
$$

$\dot{p}^{s}{ }_{i}=\left\{p_{i}^{s}, H_{T}\right\}=-\frac{\lambda}{2} \epsilon_{j i} \delta^{j k} p_{k}^{r}+\delta_{i j} r^{j}$

olarak elde edilir. Bunlardan (70) denkleminde $p_{i}^{x}$ nin tanımı yazılırsa Euler-Lagrange denklemi (37) elde edilir. 


\section{SONUÇ VE DEĞERLENDİRME}

$\mathrm{Bu}$ çalışma en genel ikinci mertebe Lagrange fonksiyonlarının (yozlaşmama şartı aramadan) birinci mertebeye indirgenmeleri ve Hamilton analizlerini sunmuştur. İndirgeme süreci 3 farklı yöntemle yapılıp karşılaştırılmıştır. Örnek olarak ise Chiral salınacı verilmiştir.

\section{TEŞEKKÜR}

Yardımlarını esirgemeyen Oğul Esen'e çok teşekkür ederim.

\section{KAYNAKLAR}

[1] Marsden, J. E. ve Ratiu, T. (1998). Introduction to mechanics ve symmetry: a basic exposition of classical mechanical systems. Springer-Verlag New York.

[2] Dirac, P.A.M. (1964). Lectures on quantum mechanics. Belfer Graduate School of Science, Monograph Series, Yeshiva University, New york.

[3] Dirac, P. A. (1958). Generalized hamiltonian dynamics. In Proceedings of the Royal Society of London A: Mathematical, Physical ve Engineering Sciences. 246(1246), 326-332.

[4] Bergmann, P. G. (1956). Quantization of general covariant field theories. Helv. Phys. Acta, Suppl. 4, 79 - 97.

[5] Gotay, M. J. ve Nester, J. M. (1979). Presymplectic Lagrangian systems. I: the constraint algorithm and the equivalence theorem. In Annales de 1'IHP Physique théorique, 30(2) ,129-142.

[6] Gotay, M. J. ve Nester, J. M. (1980). Generalized constraint algorithm ve special presymplectic manifolds. Geom. Meth. in Math. Phys., Lecture Notes in Mathematics, (775), 78-104.
[7] Gotay, M. J. Nester, J. M., ve Hinds, G. (1978). Presymplectic manifolds and the Dirac Bergmann theory of constraints. J. Math. Phys., 19(11), 2388-2399.

[8] M. Ostrogradski, (1850). Mémoires sur les équations différentielles, relatives au problème des isopérimètres. Mem. Acad. St. Petersburg VI, 4 385-517.

[9] Çağatay Uçgun F., Esen O. ve Gümral H., (2018), Reductions of topologically massive gravity I: Hamiltonian analysis of second order degenerate Lagrangians. J. Math. Phys., 59(1).

[10] Pons J. M., (1989) Ostrogradski's Theorem for Higher-Order Singular Lagrangians. Lett. Math. Phys. 17(3), 181-189.

[11] Rashid, M. S. ve Khalil, S. S. (1996). Hamiltonian description of higher order lagrangians. Int. J. of Mod. Phys. A, 11(25), 4551-4559.

[12] Schmidt, H. J. (1994). Stability ve Hamiltonian formulation of higher derivative theories. Phys. Rev. D, 49(12), 6354.

[13] Schmidt, H. J. (1995). An alternate Hamiltonian formulation of fourth-order theories ve its application to cosmology. e-print arXiv:gr-qc/9501019.

[14] Esen O. ve Guha P. (2018), On the geometry of the Schmidt-Legendre transformation. J. of Geom. Mec., 10 (3), 251291

[15] Lukierski J., Stichel P. ve Zakrzewski W., (1997) Galilean invariant $(2+1)$ dimensional models with a Chern-Simons-like term ve $\mathrm{D}=2$ noncommutative geometry. Ann. Phys. 260, 224-249.

[16] Cruz M., Gómez-Cortés R., Molgado A. ve Rojas E., (2016), Hamiltonian analysis for linearly acceleration-dependent Lagrangians. J. Math. Phys., 57, 062903. 